\title{
SIMILARITY FOR SEQUENCES OF PROJECTIONS
}

\author{
BY TOSIO KATO ${ }^{1}$
}

\author{
Communicated by C. B. Morrey, Jr., July 7, 1967
}

We consider sequences $\left\{P_{n}\right\}_{n=0,1}, \ldots$ of (not necessarily selfadjoint) projections in a Hilbert space $H$ satisfying the orthogonality conditions $P_{n} P_{m}=\delta_{m n} P_{n}$. For brevity, such a sequence $\left\{P_{n}\right\}$ will be called a $p$-sequence. A $p$-sequence $\left\{E_{n}\right\}$ is selfadjoint if $E_{n}^{*}=E_{n}$ for all $n$. A selfadjoint $p$-sequence $\left\{E_{n}\right\}$ is complete if $\sum E_{n}$, which always converges strongly, is equal to the identity.

The object of this note is to prove the following theorem.

Theorem. Let $\left\{P_{n}\right\}$ be a p-sequence, and $\left\{E_{n}\right\}$ a complete selfadjoint p-sequence. Furthermore, assume that

$$
\begin{gathered}
\operatorname{dim} P_{0}=\operatorname{dim} E_{0}=m<\infty, \\
\sum_{n=1}^{\infty}\left\|E_{n}\left(P_{n}-E_{n}\right) u\right\|^{2} \leqq c^{2}\|u\|^{2} \quad \text { for all } u \in H,
\end{gathered}
$$

where $c$ is a constant such that $0 \leqq c<1$. Then $\left\{P_{n}\right\}$ is similar to $\left\{E_{n}\right\}$, that is, there exists a nonsingular linear operator $W$ such that

$$
P_{n}=W^{-1} E_{n} W, \quad n=0,1,2, \cdots .
$$

Proof. First we shall show that

$$
W=\sum_{n=0}^{\infty} E_{n} P_{n}
$$

exists in the strong sense. Since $\sum E_{n}=1$ strongly, it suffices to show that $\sum\left(E_{n}-E_{n} P_{n}\right)=\sum E_{n}\left(E_{n}-P_{n}\right)$ converges strongly. But this is true since

$$
\left\|\sum_{n=m}^{m+p} E_{n}\left(E_{n}-P_{n}\right) u\right\|^{2}=\sum_{n=m}^{m+p}\left\|E_{n}\left(E_{n}-P_{n}\right) u\right\|^{2} \rightarrow 0, \quad m \rightarrow \infty,
$$

by (2). Incidentally, we note that (5) implies $\|A\| \leqq c<1$, where

$$
A=\sum_{n=1}^{\infty} E_{n}\left(E_{n}-P_{n}\right)=1-E_{0}-\sum_{n=1}^{\infty} E_{n} P_{n}
$$

1 This work represents part of the results obtained while the author held a Miller Research Professorship. 
Now (4) implies that $W P_{n}=E_{n} P_{n}=E_{n} W, n=0,1,2, \cdots$. Thus the theorem will be proved if we show that $W$ is nonsingular. To this end we consider

$$
W_{1}=\sum_{n=1}^{\infty} E_{n} P_{n}=1-E_{0}-A .
$$

Since $E_{0}$ is a selfadjoint projection with $\operatorname{dim} E_{0}=m<\infty, 1-E_{0}$ is a Fredholm operator with

$$
\operatorname{nul}\left(1-E_{0}\right)=m, \quad \operatorname{ind}\left(1-E_{0}\right)=0, \quad \gamma\left(1-E_{0}\right)=1,
$$

where nul $T$ denotes the nullity, ind $T$ the index, and $\gamma(T)$ the reduced minimum modulus, of the operator $T$ (for these notions see, e.g., $\left[2\right.$, Chapter IV, §5.1]). Since $\|A\|<1=\gamma\left(1-E_{0}\right)$, it follows that $W_{1}=1-E_{0}-A$ is also Fredholm, with

(8) nul $W_{1} \leqq \operatorname{nul}\left(1-E_{0}\right)=m, \quad$ ind $W_{1}=$ ind $\left(1-E_{0}\right)=0$

(see [2, Theorem 5.22]). Since

$$
W=E_{0} P_{0}+W_{1},
$$

where $E_{0} P_{0}$ is compact, $W$ is also Fredholm and ind $W=$ ind $W_{1}=0$ (see [2, Theorem 5.26]). To show that $W$ is nonsingular, it is therefore sufficient to show that nul $W=0$.

To this end we first prove that

$$
N\left(W_{1}\right)=P_{0} H,
$$

where $N(T)$ denotes the null space of $T$. In fact, we have $W_{1} P_{0}=0$ by (7) so that $N\left(W_{1}\right) \supset P_{0} H$. But since $\operatorname{dim} P_{0}=m$ and nul $W_{1} \leqq m$ by (8), we must have (10).

Suppose now that $W u=0$. Then $0=E_{0} W u=E_{0} P_{0} u$ and $W_{1} u=W u$ $-E_{0} P_{0} u=0$. Hence $u=P_{0} u$ by $(10)$ and so $E_{0} u=E_{0} P_{0} u=0$. Thus $(1-A) u=\left(W_{1}+E_{0}\right) u=0$ by (7). Since $\|A\|<1$, we obtain $u=0$. This shows that nul $W=0$ and completes the proof.

REMARK. It has been shown by C. Clark [1] that the theorem is useful in proving that certain ordinary differential operators are spectral in the sense of Dunford.

\section{BIBLIOGRAPHY}

1. C. Clark, On relatively bounded perturbations of ordinary differential operators, Pacific J. Math. (to appear)

2. T. Kato, Perturbation theory for linear operators, Springer, Berlin, 1960.

University of California, Berkeley 